\title{
Clinicopathological features of dermatofibrosarcoma protuberans
}

\author{
NOPPADOL LARBCHAROENSUB ${ }^{1}$, JITCHAI KAYANKARNNAVEE ${ }^{1}$, SUDA SANPAPHANT ${ }^{1}$, \\ KIDAKORN KIRANANTAWAT ${ }^{2}$, CHEWARAT WIROJTANANUGOON ${ }^{3}$ and VORACHAI SIRIKULCHAYANONTA ${ }^{1}$
}

Departments of ${ }^{1}$ Pathology, ${ }^{2}$ Surgery and ${ }^{3}$ Radiology, Faculty of Medicine Ramathibodi Hospital, Mahidol University, Bangkok 10400, Thailand

Received November 8, 2014; Accepted August 25, 2015

DOI: $10.3892 / \mathrm{ol} .2015 .3966$

\begin{abstract}
Dermatofibrosarcoma protuberans (DFSP) is a superficial cutaneous tumor of low malignant potential characterized by a high rate of local recurrence. The histopathological appearance shows uniform spindle neoplastic cells arranged in a predominantly storiform pattern, typically with positive staining for cluster of differentiation (CD)34 and vimentin on immunohistochemistry. A minority of cases of DFSP have areas of sarcomatous transformation. Wide surgical excision is the cornerstone of treatment for DFSP. The objective of the present study was to determine the clinicopathological features of DFSP. Pathological records were searched for cases of DFSP in the database of the Department of Pathology, Faculty of Medicine Ramathibodi Hospital (Mahidol University, Bangkok, Thailand) between 1994 and 2013. The results showed 68 cases with DFSP. The mean age at diagnosis was 40 years (range, 3-86 years). Among this group of patients, 26 cases $(38.2 \%)$ experienced local recurrence and $6(8.8 \%)$ exhibited sarcomatous transformation of DFSP. The factors that predict the recurrence of DFSP are an incorrect first pathological diagnosis and an inadequate surgical margin. The factors that predict the sarcomatous transformation of DFSP are a larger tumor size and an incorrect first pathological diagnosis. In patients who have tumors with spindle cells arranged in a storiform pattern, CD34 immunohistochemical staining provides the definitive diagnosis. Exact histopathological categorization is important to select the appropriate treatment and predict the clinical outcome.
\end{abstract}

Correspondence to: Dr Noppadol Larbcharoensub, Department of Pathology, Faculty of Medicine Ramathibodi Hospital, Mahidol University, 270 Rama VI Road, Ratchathewi, Bangkok 10400, Thailand

E-mail: noppadol.lar@mahidol.ac.th

Key words: dermatofibrosarcoma protuberans, sarcomatous transformation, recurrence, aggressive cutaneous tumor

\section{Introduction}

Dermatofibrosarcoma protuberans (DFSP) is a superficial, low-grade, locally aggressive, spindle, fibroblastic, neoplastic lesion. As a relatively uncommon neoplasm and locally aggressive cutaneous tumor, it is characterized by high rates of local recurrence, but a low risk of metastasis (1-4). DFSP typically presents with a purple or pink asymptomatic plaque or nodule, with a history of slow but persistent growth (1). DFSPs usually affect young to middle-aged adult patients (1-4). The tumor was first described in 1924 by Darier and Ferrand as a 'progressive and recurring dermatofibroma', which is a nodular cutaneous tumor characterized by a prominent storiform pattern (5). In addition to the classical form characterized by a storiform pattern of tumor cells, the pigmented (Bednar's tumor), plaque-like and myxoid types, and DFSP with sarcomatous areas can be observed (1-4). Classical DFSP and Bednar's tumors are readily diagnosed, however, the myxoid type represents a challenge diagnostically. Immunoreactivity for cluster of differentiation (CD) 34 in a spindle cell tumor is the main immunohistochemical marker for the diagnosis of DFSP (1-4,6,7). Although DFSP has been regarded as a low-grade sarcoma, certain cases have sarcomatous transformation, which is characterized by hypercellular spindle cell fascicles with increased atypia, mitosis tumor necrosis and usually, the loss of CD34 immunoreactivity $(1,3,4)$.

The objective of the present study was to analyze the correlation between clinical and pathological factors, including age, gender, tumor size, anatomical location, mitotic counts, surgical margin, recurrence and high-grade sarcomatous transformation, in a large series of patients with DFSP from a single center.

\section{Materials and methods}

Patients and criteria. A retrospective study was performed on DFSP cases diagnosed by surgical specimens from wide excisional biopsies obtained from the Department of Pathology, Faculty of Medicine Ramathibodi Hospital (Mahidol University, Bangkok, Thailand) over a period of 20 years, between 1994 and 2013. The histopathological diagnosis of DFSP was reviewed. The criteria for the diagnosis of DFSP were: i) The presence of spindle cells irregularly organized in linked fascicles with a storiform arrangement, 
and histopathological features compatible with DFSP; and ii) positive CD34 and vimentin immunohistochemical staining in spindle tumor cells. Information obtained from the medical records, including patient's age at first diagnosis, gender, smoking history, tumor size, surgical margin of tumor, anatomical location, first pathological diagnosis, mitotic counts, modality of treatment, surgical margin, recurrence and treatment outcomes, were collected and analyzed. Patients were grouped based on the sarcomatous transformation or recurrence of the tumor.

Statistical analysis. A comparison between the clinicopathological features was evaluated using the chi-square test. Univariate log-rank analysis was performed. Two-tailed Fisher's exact test was used to evaluate statistical significance between the groups. $\mathrm{P}<0.05$ was considered to indicate a statistically significant difference. All statistical analyses were performed using SPSS software, version 13.0 (SPSS Inc, Chicago, IL, USA).

Study approval. This study was approved by the Committee on Human Rights related to research involving human subjects of Faculty of Medicine Ramathibodi Hospital, Mahidol University (ID 11-54-33).

\section{Results}

Patient and tumor characteristics, and treatment modalities. In total, 68 cases consisting of 32 male and 36 female patients, with an age range of 3-86 years old, and a mean and median age of 40 and 39 years, respectively, met the inclusion criteria. All patients presented with primary disease without evidence of metastasis. The characteristics of the DFSP patients are summarized in Table I. The anatomical locations of the lesions were as follows: Head and neck, 19 cases; upper extremities, 9 cases; lower extremities, 18 cases; body, 20 cases; and genitalia, 2 cases. The tumor sizes ranged from $0.2-10 \mathrm{~cm}$ (mean, $3.2 \pm 2.3 \mathrm{~cm}$; median, $2.5 \mathrm{~cm}$ ). The most common first pathological diagnosis was DFSP (53 patients; $77.9 \%$ ), while a spindle cell tumor was the first diagnosis in 4 patients $(5.9 \%)$. Therefore, the overall sensitivity for diagnosis was $83.8 \%$. The false-negative initial diagnoses included dermatofibroma (5 cases), neurofibroma (4 cases), fibromatosis and schwannoma (1 case each). There were 62 cases of DFSP without sarcomatous transformation and 6 cases of sarcomatous transformation in DFSP. A total of 62 patients underwent a wide excision only, 4 patients underwent a wide excision with radiotherapy (4500-7000 cGy), 1 patient underwent a wide excision with radiotherapy followed by chemotherapy (Adriamycin for undifferentiated sarcoma arising in DFSP) and 1 patient underwent a wide excision with imatinib targeted therapy ( $800 \mathrm{mg}$ daily for 4 months). Of the 6 cases with sarcomatous transformation, 4 underwent a wide excision only. The study also noted 1 patient who developed distant pulmonary metastasis.

Sarcomatous transformation. Of the 68 patients with DFSP, 6 developed sarcomatous transformation. The characteristics of sarcomatous transformation in the DFSP patients are summarized in Table II. Case 1 underwent surgery 19 times,
Table I. Clinicopathological characteristics of DFSP patients.

\begin{tabular}{lrr}
\hline Characteristics & Cases, $\mathrm{n}$ & $\%$ \\
\hline Gender & & \\
Male & 32 & 47.1 \\
Female & 36 & 52.9 \\
Smoking (n=48) & & \\
Yes & 5 & 10.4 \\
No & 43 & 89.6 \\
Anatomical location & & \\
Head and neck & 19 & 27.9 \\
Upper extremities & 9 & 13.2 \\
Lower extremities & 18 & 26.5 \\
Body & 20 & 29.4 \\
Genitalia & 2 & 2.9 \\
First pathological diagnosis & & \\
DFSP & 53 & 77.9 \\
Dermatofibroma & 5 & 7.4 \\
Neurofibroma & 4 & 5.9 \\
Spindle cell tumor & 4 & 5.9 \\
Schwannoma & 1 & 1.5 \\
Fibromatosis & 1 & 1.5 \\
Recurrence & &
\end{tabular}

Recurrence

Yes 26

38.2

No $\quad 42$

61.8

Number of surgeries

1

73.5

2

50

17.6

3

4.4

$\geq 4$

4.4

Sarcomatous transformation

$\begin{array}{lrr}\text { Yes } & 6 & 8.8\end{array}$

No 62

91.2

Modality of treatment

$\begin{array}{lll}\text { Wide excision only } & 62 & 91.1\end{array}$

Wide excision with radiotherapy $\quad 4 \quad 5.9$

Wide excision with radiotherapy $\quad 1 \quad 1.5$

and chemotherapy

Wide excision with imatinib $\quad 1 \quad 1.5$

targeted therapy

Surgical margin $(n=45)$

Free $(\geq 1 \mathrm{~cm})$

11

24.4

Not free $(<1 \mathrm{~cm})$

34

75.6

DFSP, dermatofibrosarcoma protuberans.

with 18 surgeries for recurrence at the head and neck region since the time of first diagnosis at the age of 41 years. The surgical resected margin was not disease-free and the tumor invaded the periosteum, pericranium and bony structures (Fig. 1). The tumor showed a classical area of spindle tumor cells arranged in a storiform pattern, with focal active mitoses [20/10 high-power fields (HPF)] (Fig. 2). The patient 

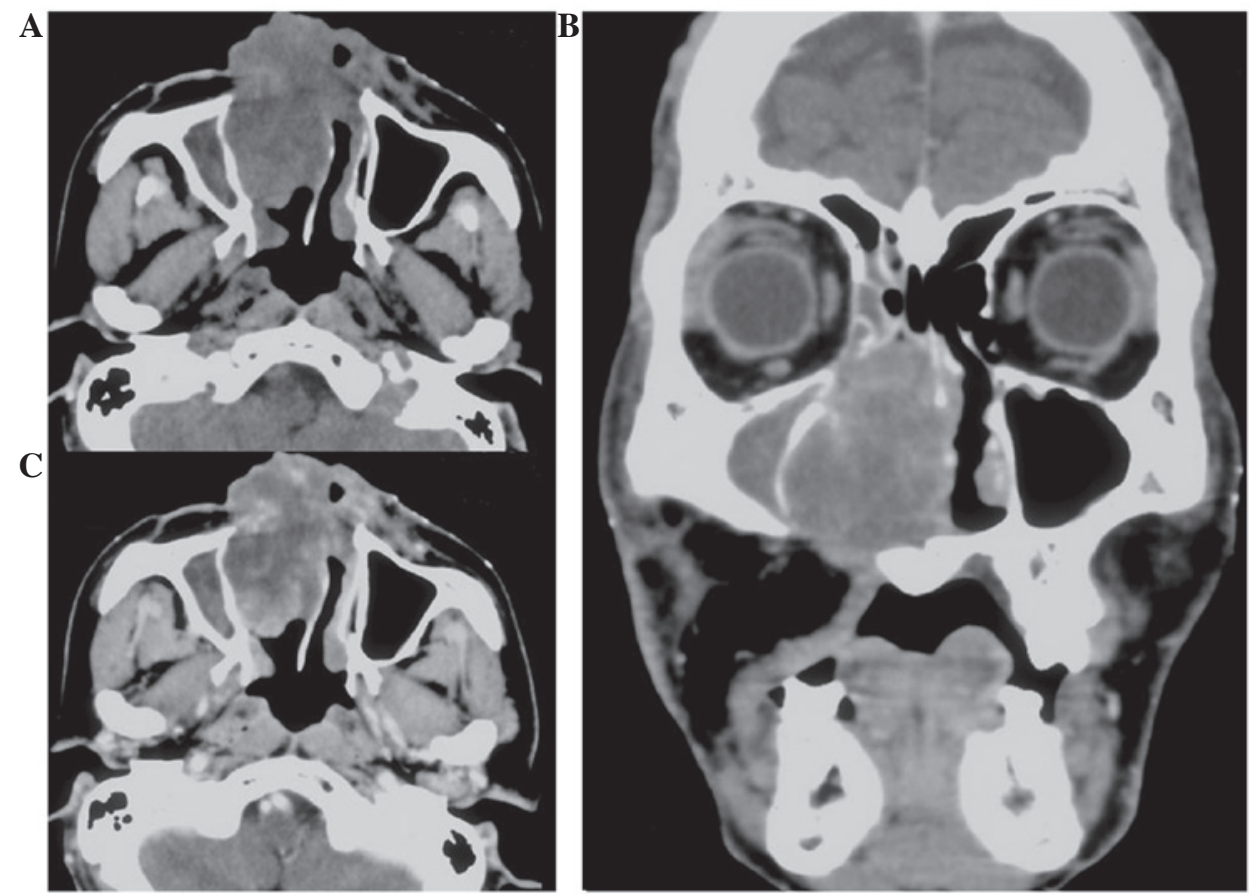

Figure 1. (A) Non-enhanced axial CT, and contrast-enhanced (B) coronal and (C) axial CT images showing a well-defined, lobulated, hypodense, soft-tissue mass, with heterogeneous enhancement involving the skin and subcutaneous tissue of the nose. Involvement extended into the nasal cavities, more on the right side, with associated bony destruction at the nasal septum. CT, computed tomography.
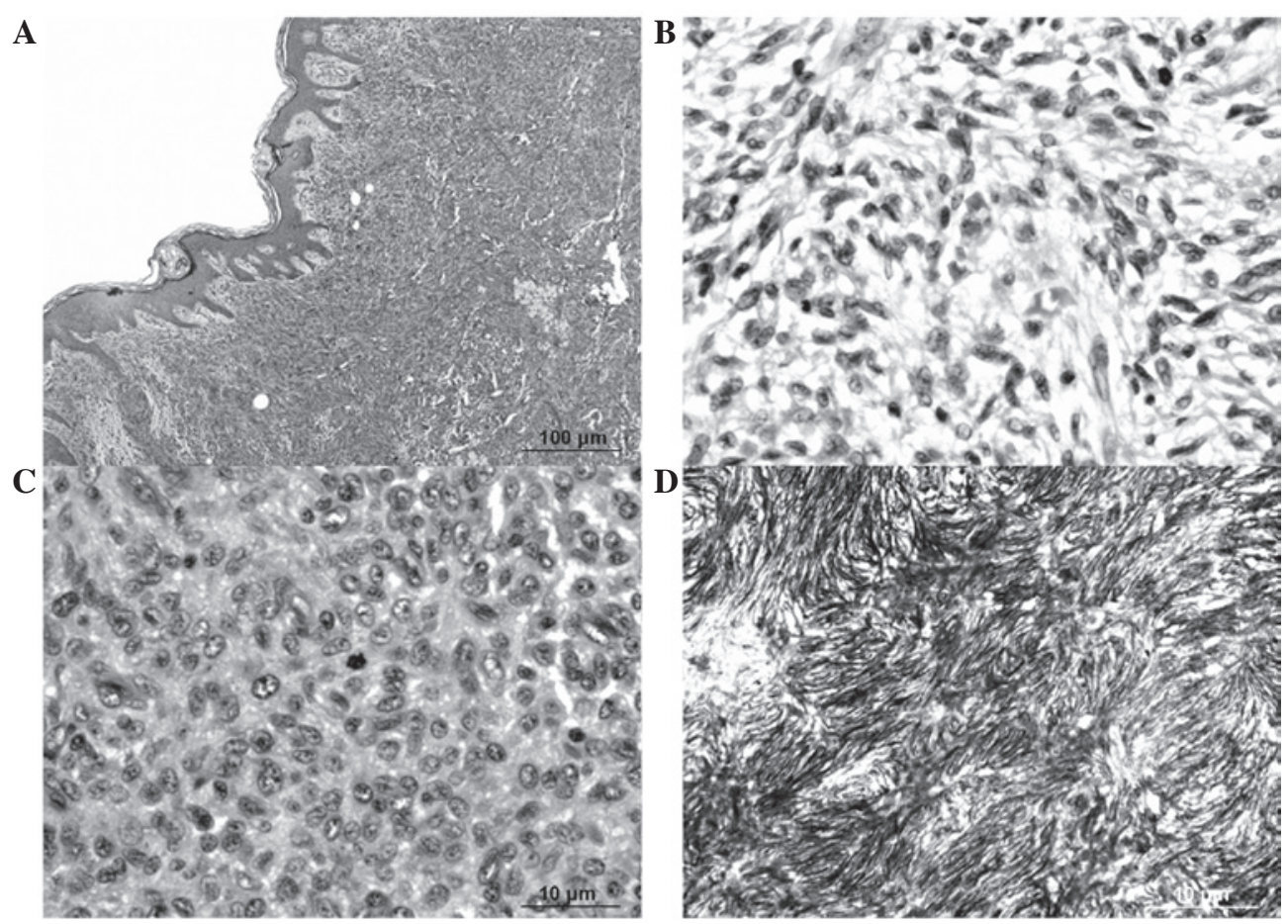

Figure 2. Histopathology revealing a classical area of spindle tumor cells arranged in a storiform pattern [(A) $\mathrm{x} 40$ and (B) $\mathrm{x} 400 \mathrm{magnification}$; H\&E], with (C) focal active mitoses (H\&E; x400 magnification). (D) Immunohistochemistry showing positive staining for cluster of differentiation 34 (x400 magnification). H\&E, hematoxylin and eosin.

developed secondary transformation of malignant fibrous histiocytoma (MFH) after receiving 18 surgical resections. The patient subsequently developed pulmonary metastasis and finally succumbed to the disease 20 years after the initial diagnosis of DFSP and 17 months after the diagnosis of MFH. No autopsy was performed.
Case 2 experienced tumor recurrence at the lower extremity, although the surgical margin used was $1 \mathrm{~cm}$. Mitotic activity was 11/10 HPF. Case 3 experienced undifferentiated sarcomatous transformation of the tumor at the lower extremity. The mitotic rate was 10/10 HPF. The patient underwent a below-knee amputation and received $6500 \mathrm{cGy}$ 
Table II. Summary of patients with sarcomatous transformation in DFSP.

\begin{tabular}{|c|c|c|c|c|c|c|c|c|c|}
\hline $\begin{array}{l}\text { Case } \\
\text { no. }\end{array}$ & Gender & $\begin{array}{l}\text { Age, } \\
\text { years }\end{array}$ & $\begin{array}{l}\text { Tumor } \\
\text { size, cm }\end{array}$ & $\begin{array}{l}\text { Margin, } \\
\mathrm{cm}\end{array}$ & Location & $\begin{array}{l}\text { Mitosis, } \\
\text { /10 HPF }\end{array}$ & $\begin{array}{c}\text { First } \\
\text { pathological } \\
\text { diagnosis }\end{array}$ & $\begin{array}{c}\text { Type of } \\
\text { sarcomatous } \\
\text { transformation }\end{array}$ & $\begin{array}{c}\text { No. of } \\
\text { surgeries }\end{array}$ \\
\hline 1 & Male & 41 & 7.5 & 0 & Head and neck & 20 & Neurofibroma & MFH & 19 \\
\hline 2 & Male & 46 & 1.5 & 1 & Lower extremity & 11 & Neurofibroma & Fibrosarcoma & 2 \\
\hline 3 & Male & 37 & 7.5 & $<1$ & Lower extremity & 10 & Dermatofibroma & $\begin{array}{l}\text { Undifferentiated } \\
\text { sarcoma }\end{array}$ & 5 \\
\hline 4 & Female & 86 & 3.2 & 0.1 & Lower extremity & 5 & DFSP & MFH & 2 \\
\hline 5 & Female & 33 & 7 & 0.1 & Upper extremity & 5 & DFSP & Fibrosarcoma & 4 \\
\hline 6 & Male & 39 & 6.5 & 2 & Body & 10 & DFSP & Fibrosarcoma & 2 \\
\hline
\end{tabular}

HPF, high-power fields; DFSP, dermatofibrosarcoma protuberans; MFH, malignant fibrous histiocytoma.

Table III. Correlation between clinicopathological features and sarcomatous transformation in DFSP.

\begin{tabular}{|c|c|c|c|}
\hline Characteristics & $\operatorname{DFSP}(n=62)$ & $\begin{array}{l}\text { DFSP with sarcomatous } \\
\text { transformation }(n=6)\end{array}$ & P-value \\
\hline Age, years ${ }^{\mathrm{a}}$ & $39.27 \pm 15.64(3-70)$ & $47.00 \pm 19.59(33-86)$ & 0.262 \\
\hline Gender, $\mathrm{n}$ & & & 0.410 \\
\hline Male & 28 & 4 & \\
\hline Female & 34 & 2 & \\
\hline Tumor size, $\mathrm{cm}^{\mathrm{a}}$ & $2.97 \pm 2.17(0.2-10)$ & $5.53 \pm 2.55(1.5-7.5)$ & 0.008 \\
\hline Anatomical location, $\mathrm{n}$ & & & 0.682 \\
\hline Head and neck & 18 & 1 & \\
\hline Upper extremity & 8 & 1 & \\
\hline Lower extremity & 15 & 3 & \\
\hline Body & 19 & 1 & \\
\hline Genitalia & 2 & 0 & \\
\hline Histopathological subtype, $\mathrm{n}$ & & & 0.075 \\
\hline Conventional & 52 & 4 & \\
\hline Myxoid & 3 & 2 & \\
\hline Plaque-like & 3 & 0 & \\
\hline Bednar & 4 & 0 & \\
\hline First pathological diagnosis, $n$ & & & 0.049 \\
\hline True & 54 & 3 & \\
\hline False & 8 & 3 & \\
\hline Recurrence, $\mathrm{n}$ & & & 0.145 \\
\hline No & 40 & 2 & \\
\hline Yes & 22 & 4 & \\
\hline Surgical margin $(n=45)$ & & & 0.150 \\
\hline Free $(\geq 1 \mathrm{~cm})$ & 9 & 2 & \\
\hline Not free $(<1 \mathrm{~cm})$ & 30 & 4 & \\
\hline
\end{tabular}

${ }^{a}$ Mean \pm standard deviation (range). Significant values $(\mathrm{P}<0.05)$ are shown in bold. DFSP, dermatofibrosarcoma protuberans.

radiotherapy followed by adjuvant chemotherapy with 4 cycles of adriamycin $\left(75 \mathrm{mg} / \mathrm{m}^{2}\right)$. Case 4 was the oldest patient in this study, who developed MFH transformation in DFSP. The patient received radiotherapy with a total dose of 4,500 cGy. Case 5 was the youngest patient (33 years) in the group of patients with sarcomatous transformation in DFSP. The last case, case 6, was a patient with fibrosarcomatous transformation in DFSP, which presented with a rapidly growing mass on the right chest wall. The remaining 4 patients (cases 1,2,5 and 6) received only surgical wide excision. 
Table IV. Correlation between clinicopathological features and recurrence of DFSP.

\begin{tabular}{|c|c|c|c|}
\hline Characteristics & $\begin{array}{l}\text { DFSP without } \\
\text { recurrence }(n=42)\end{array}$ & $\begin{array}{l}\text { DFSP with } \\
\text { recurrence }(n=26)\end{array}$ & P-value \\
\hline Age, years $^{\mathrm{a}}$ & $39.07 \pm 16.86(3-86)$ & $41.38 \pm 14.73(4-70)$ & 0.566 \\
\hline Gender, $\mathrm{n}$ & & & 0.129 \\
\hline Male & 17 & 15 & \\
\hline Female & 25 & 11 & \\
\hline Tumor size, $\mathrm{cm}^{\mathrm{a}}$ & $2.82 \pm 2.26(0.2-10)$ & $3.81 \pm 2.28(0.6-9)$ & 0.086 \\
\hline Anatomical location, $\mathrm{n}$ & & & 0.061 \\
\hline Head and neck & 8 & 11 & \\
\hline Upper extremity & 7 & 2 & \\
\hline Lower extremity & 12 & 6 & \\
\hline Body & 15 & 5 & \\
\hline Genital organ & 0 & 2 & \\
\hline Histopathological subtype, $\mathrm{n}$ & & & 0.119 \\
\hline Conventional & 35 & 21 & \\
\hline Myxoid & 1 & 4 & \\
\hline Plaque-like & 3 & 0 & \\
\hline Bednar & 3 & 1 & \\
\hline First pathological diagnosis, $\mathrm{n}$ & & & $<0.001$ \\
\hline True & 42 & 15 & \\
\hline False & 0 & 11 & \\
\hline Sarcomatous transformation, $\mathrm{n}$ & & & 0.193 \\
\hline No & 40 & 22 & \\
\hline Yes & 2 & 4 & \\
\hline Surgical margin $(n=45)$ & & & 0.042 \\
\hline Free $(\geq 1 \mathrm{~cm})$ & 8 & 3 & \\
\hline Not free $(<1 \mathrm{~cm})$ & 16 & 18 & \\
\hline
\end{tabular}

${ }^{a}$ Mean \pm standard deviation (range). Significant values $(\mathrm{P}<0.05)$ are shown in bold. DFSP, dermatofibrosarcoma protuberans.

The univariate log-rank analysis identified a large tumor size and an incorrect first pathological diagnosis as significant parameters associated with sarcomatous transformation in DFSP. The mean size of the tumor with DFSP and sarcomatous transformation was $5.53 \mathrm{~cm}$ (range, $1.5-7.5 \mathrm{~cm}$ ), which was significantly larger than the mean size of the tumor in the DFSP patients without sarcomatous transformation $(2.97 \mathrm{~cm}$; range, $0.2-10 \mathrm{~cm})(\mathrm{P}=0.008)$. The false-negative first pathological diagnosis of DFSP also showed significant correlation with sarcomatous transformation in DFSP $(\mathrm{P}=0.049)$. As shown in Table III, no statistically significant correlation was found between sarcomatous transformation and age, gender, anatomical location, histopathological subtype, recurrence and surgical margin (at $1 \mathrm{~cm}$ ).

Outcome and recurrence. The patients underwent treatment as described. In total, 26 cases experienced recurrence. The head and neck region (11 cases) was the most common anatomical location for tumor recurrence. The body and extremities exhibited lower risks of recurrence. As shown in Table IV, no statistically significant correlation was found between recurrent DFSP and age, gender, tumor size, anatomical location, histopathological subtype and sarcomatous transformation. Conventional surgery was adopted in limited sites where a wide excision would have been difficult to perform, including the head and neck region, and genitalia $(n=21)$, which showed a significant association with recurrent DFSP $(\mathrm{P}=0.028)$. Furthermore, the incorrect first pathological diagnosis remained a highly significant prognostic factor of the sarcomatous transformation and recurrence of DFSP $(\mathrm{P}=0.049$ and $\mathrm{P}<0.001$, respectively).

\section{Discussion}

In the current World Health Organization classification, DFSP is defined as a superficial, low-grade, locally aggressive fibroblastic neoplasm $(1,2)$. The present study retrospectively collected data on DFSP that was uniformly diagnosed based on the expression of CD34 and vimentin by immunohistochemistry in a single medical institution. DFSP is a rare disease with a favorable prognosis and relatively low mortality $(1,2)$. In the present study, the patient's ages ranged from 3-86 years. The anatomical locations that were mostly affected were the body $(n=20)$, followed by the head and neck $(n=19)$, and the 
lower extremities $(n=18)$. The lower extremities were more commonly affected than the upper extremities. These findings are in agreement with the majority of previous studies in the literature (1-4). The first pathological diagnosis of almost all tumors was DFSP. The overall sensitivity for the histopathological diagnosis of DFSP was $83.8 \%$. A total of 11 patients were diagnosed firstly with other pathologies, including dermatofibroma in 5 cases, neurofibroma in 4 cases, fibromatosis in 1 case and schwannoma in 1 case. The components of these tumors are composed of spindle cells, which can mimic DFSP and its variants, particularly the myxoid histopathological subtype. Once pathologists re-evaluated the tumor and additional CD34 immunohistochemical study was performed, the pathological diagnosis was changed to DFSP.

The immunohistochemical markers identified for DFSP are CD34 and vimentin (1-4). However, CD34 immunohistochemical positivity is also expressed by other spindle cell tumors, including solitary fibrous tumors, sclerotic fibroma, superficial acral fibromyxoma, cellular digital fibroma, dermatofibroma and nuchal-type fibroma. Additional immunohistochemical staining of factor XIIIa, stromelysin III, CD44, CD163 and D2-40 have been found to be positive in dermatofibroma and negative in DFSP (1-4,6-10). Spindle tumor cells are immunonegative for S100 protein, smooth muscle actin, desmin, cytokeratin and epithelial membrane antigen (1-4).

Wide excision remains the cornerstone of the treatment of DFSP (10-12). However, the response from patients remains poor and locoregional recurrence has been observed during follow-up (10-12). The natural history of DFSP is low-grade malignancy, with a 26-60\% local recurrence rate attributed to incomplete excision due to poor circumscription and irregular boundaries (13). The lesion typically infiltrates well beyond its grossly visible margin into the surround tissue. Certain studies have advocated the use of Mohs micrographic surgery with incremental excision until normal tissue is obtained, as documented by repeat frozen sections (13). Several studies have demonstrated a significant correlation between a wide excision and a low recurrence rate $(9,13)$. In the present study, the recurrence of DFSP occurred in 26 cases (38.2\%). Locoregional recurrence after such excision appears to be associated with anatomical tumor location, adequacy of surgical margin and a corrected first pathological diagnosis. The main site of tumor recurrence was mostly the head and neck, which is the common site of recurrent tumor in this study. We hypothesize that this may be due to the difficulty of performing a resection with free surgical margins in this anatomical location. An adequate surgical margin cannot be completely obtained in these regions. The periosteum, pericranium and cervical fascia of the head and neck are the limited anatomical sites for complete wide surgical excision. In conclusion, the major factor predicts the recurrence of tumor was inadequate surgical margin.

An incorrect first pathological diagnosis was significantly associated with sarcomatous transformation and the recurrence of DFSP $(\mathrm{P}=0.049$ and $\mathrm{P}<0.001$, respectively). DFSP is a distinct tumor entity that often presents a diagnostic challenge. In the present study, the false-negative pathological diagnoses included dermatofibroma, neurofibroma, schwannoma and fibromatosis, which are all benign conditions. Initial misdiagnosis led to a delay in treatment, with the tumor producing significant local extension and destruction. An incorrect first pathological diagnosis may lead to a negative morbidity outcome as serious as failure to treat a missed case of low-grade sarcoma, DFSP. Successful DFSP treatment begins with an accurate pathological diagnosis.

Sarcomatous change in DFSP represents a form of tumor progression, which occurs in 10-15\% of DFSP and is associated with a prognosis worse than ordinary DFSP (1,14-18). In the present study, sarcomatous transformation occurred in $6 / 68(8.8 \%)$ of DFSP cases. These sarcomatous transformations included fibrosarcoma (3/6), followed by MFH $(2 / 6)$ and undifferentiated sarcoma (1/6). The factors predicting sarcomatous transformation in this study were a large tumor size and an incorrect first pathological diagnosis $(\mathrm{P}=0.008$ and $\mathrm{P}=0.049$, respectively).

The characteristic cytogenetic features of DFSP are a supernumerary ring chromosome and a reciprocal chromosomal translocation $\mathrm{t}(17 ; 22)(\mathrm{q} 22 ; \mathrm{q} 13)$, causing a fusion of the platelet-derived growth factor $\beta$-chain $(P D G F B)$ gene at $22 \mathrm{q} 13$ and the collagen type $1 \alpha 1(C O L 1 A 1)$ at $17 q 22(1)$. This rearrangement results in the constitutive activation of the PDGF as a consequence of deregulated ligand expression (19). $P D G F B$ copy number status may become a useful diagnostic marker since the gene is a potential target of treatment in patients with DFSP. Imatinib, the target therapy for $P D G F B$, was administered to 1 patient in the present study for 4 months. The patient experienced disease-free survival for $>103$ months. Further molecular study in DFSP patients is warranted and has important implications for the study of the pathogenesis of disease.

In conclusion, the present study found that the factors that predict the sarcomatous transformation of DFSP are a larger tumor size and an incorrect first pathological diagnosis. The factors that predict the recurrence of DFSP are an incorrect first pathological diagnosis and an inadequate surgical margin. In patients who have the spindle cell tumors arranged in a storiform pattern, CD34 immunohistochemical staining provides the pathological diagnosis of DFSP. However, a combination of clinicopathological features, immunohistochemistry and, in specific cases, molecular or cytogenetic testing, is essential for definitive diagnosis. The exact histopathological categorization is important for selecting the appropriate treatment and for predicting the clinical outcome.

\section{References}

1. Mentzel T, Peeutour F, Lazar A and Coindre JM: Dermatofibrosarcoma protuberans. In: World Health Organization (WHO) Classification of Tumours of Soft tissue and Bone. Pathology and Genetics. Fletcher CDM, Bridge JA, Hogendoorn P and Martens F (eds). Vol 5. 4th edition. IARC Press, Lyon, pp77-79, 2013.

2. Weyers W, Mentzel T, Kasper RC, Tosti A, Iorizzo M, Zelger B and Caputo R: In: World Health Organization Classification of Tumours. Pathology and Genetics Skin Tumours. Fibrous, fibrohistiocytic and histiocytic tumours. LeBoit PE, Burg G, Weedon D and Sarasin A (eds). IARC Press, Lyon, pp259-261, 2002.

3. Kempson RL, Fletcher CDM, Evans HL, Hendrickson MR and Sibley RK: Atlas of Tumor Pathology. Tumors of Soft Tissues. 3rd series. Fascicle 30. Armed Forces Institute of Pathology, Washington, DC, pp138-148, 2001.

4. Goldblum JR, Folpe AL and Weiss SW (eds): Fibrohistocytic tumors of intermediate malignancy. In: Enzinger and Weiss's soft tissue tumors. 6th edition. Elsevier Saunders, Philadelphia, PA, pp387-400, 2013. 
5. Darier J and Ferrand M: Dermatofibromes progressifs et récidivants ou fibrosarcomes de la peau. Ann Dermatol Syphiligr (Paris) 5: 545-562, 1924.

6. Prieto VG, Reed JA and Shea CR: CD34 immunoreactivity distinguishes between scar tissue and residual tumor in re-excisional specimens of dermatofibrosarcoma protuberans. J Cutan Pathol 21: 324-329, 1994.

7. Llombart B, Serra-Guillén C, Monteagudo C, López Guerrero JA and Sanmartín O: Dermatofibrosarcoma protuberans: A comprehensive review and update on diagnosis and management. Semin Diagn Pathol 30: 13-28, 2013.

8. Erdem O, Wyatt AJ, Lin E, Wang $X$ and Prieto VG: Dermatofibrosarcoma protuberans treated with wide local excision and followed at a cancer hospital: Prognostic significance of clinicopathologic variables. Am J Dermatopathol 34: 24-34, 2012.

9. Stivala A, Lombardo GA, Pompili G, Tarico MS, Fraggetta F and Perrotta RE: Dermatofibrosarcoma protuberans: Our experience of 59 cases. Oncol Lett 4: 1047-1055, 2012.

10. Archontaki M, Korkolis DP, Arnogiannaki N, Konstantinidou C, Georgopoulos S, Dendrinos P, Zarkadas G and Kokkalis G: Dermatofibrosarcoma protuberans: A case series of 16 patients treated in a single institution with literature review. Anticancer Res 30: 3775-3779, 2010.

11. Fiore M, Miceli R, Mussi C, Lo Vullo S, Mariani L, Lozza L, Collini P, Olmi P, Casali PG and Gronchi A: Dermatofibrosarcoma protuberans treated at a single institution: A surgical disease with a high cure rate. J Clin Oncol 23: 7669-7675, 2005.
12. DuBay D, Cimmino V, Lowe L, Johnson TM and Sondak VK: Low recurrence rate after surgery for dermatofibrosarcoma protuberans: A multidisciplinary approach from a single institution. Cancer 100: 1008-1016, 2004.

13. Lemm D, Mügge LO, Mentzel T and Höffken K: Current treatment options in dermatofibrosarcoma protuberans. J Cancer Res Clin Oncol 135: 653-665, 2009.

14. Connelly JH and Evans HL: Dermatofibrosarcoma protuberans. A clinicopathologic review with emphasis on fibrosarcomatous areas. Am J Surg Pathol 16: 921-925, 1992.

15. Sato N, Kimura K and Tomita Y: Recurrent dermatofibrosarcoma protuberans with myxoid and fibrosarcomatous changes paralleled by loss of CD34 expression. J Dermatol 22: 665-672, 1995.

16. Mentzel T, Beham A, Katenkamp D, Dei Tos AP and Fletcher CD: Fibrosarcomatous ('high-grade') dermatofibrosarcoma protuberans: Clinicopathologic and immunohistochemical study of a series of 41 cases with emphasis on prognostic significance. Am J Surg Pathol 22: 576-587, 1998.

17. Goldblum JR, Reith JD and Weiss SW: Sarcomas arising in dermatofibrosarcoma protuberans: A reappraisal of biologic behavior in eighteen cases treated by wide local excision with extended clinical follow up. Am J Surg Pathol 24: 1125-1130, 2000.

18. Abbott JJ, Oliveira AM and Nascimento AG: The prognostic significance of fibrosarcomatous transformation in dermatofibrosarcoma protuberans. Am J Surg Pathol 30: 436-443, 2006.

19. McArthur G: Dermatofibrosarcoma protuberans: Recent clinical progress. Ann Surg Oncol 14: 2876-2886, 2007. 